\title{
Integrated EMC Education at San Francisco State University
}

\author{
Zorica Pantic-Tanner \\ School of Engineering \& Computer Science \\ San Francisco State University, CA 94132
}

\begin{abstract}
This paper describes how EMC principles are integrated throughout the undergraduate curriculum at San Francisco State University (SFSU). With increase in clock frequencies and data rates in digital circuits, as well as with a push toward microwave and higher frequency ranges in communication systems, the problems of electromagnetic interference are becoming more pronounced. It is imperative for graduating engineers to have operative knowledge of EMI and corresponding remedies. The EMC-related principles and applications are incorporated in two electromagnetics, one communications and one EMC course and are supported by hands-on experience in a newly developed state-of-the-art high-frequency laboratory. The lab exercises and design projects are described at the web site http://http.engineering.sfsu.edu/nsf/. They help students understand the difficult EM/EMC concepts and expose them to practical EMC applications.
\end{abstract}

\section{Introduction}

In this era of rapid development of communication systems, the trend is toward use of microwave and higher frequency ranges. At the same time circuits and systems are operating at higher data rates, and becoming smaller and more compact. As a result, the environment is rapidly becoming saturated with broadband electromagnetic noise that can be easily coupled to a circuit/system through electric, magnetic or electromagnetic mechanisms. Designing circuits and systems that are electromagnetically compatible, i.e., do not interfere with the other systems and are not susceptible to electromagnetic noise, is becoming more and more challenging. It is necessary for every practicing electrical engineer to be aware of these problems. Undergraduate students should be exposed to electromagnetic compatibility (EMC) concepts and learn about possible solutions.

SFSU is one of few universities nationwide that incorporates the EMC principles and solutions into undergraduate curriculum and has also developed an active undergraduate research program in the EMC area. The theoretical principles are integrated in two electromagnetics, one communications and one undergraduate EMC course, and are supported by hands-on experience in a state-of-the-art EMC/Communications laboratory. Students capstone design projects can also contain an EMC component. The seed money for the EMC curriculum development was funded by the Santa Clara Valley (SCV) chapter of the IEEE 
EMC Society in 1993. The IEEE grant was used to establish and teach an undergraduate EMC course that included both theoretical and experimental part. Some of the experiments were performed in the existing SFSU Communication Systems Laboratory but the others, due to the lack of adequate equipment, were hosted by some of the Silicon Valley companies. Overall, the course was a great success and prompted the IEEE EMC Society to establish an international University Grant Program for EMC curriculum development. The further SFSU EMC laboratory development was in part funded by the National Science Foundation Grant DUE 9751722 and in part by generous equipment donations from companies such as Hewlett Packard, Silicon Graphics, Lindgren RF Enclosures and Sony Electronics. The lab currently has state-of-the-art spectrum analyzers, network analyzers, immunity equipment and a GTEM Cell for making precision emission and immunity measurements. Numerical modeling tools include the FiniteElement-Method (FEM), Method-Of-Moments (MOM), and Finite-Difference-Time-Domain (FDTD) solvers.

The NSF grant was also used to develop eleven new lab experiments and design projects with EMC applications. They vary from non-ideal behavior of circuit components, probing of EM noise sources such as power supplies and printed circuit boards (PCBs), surface and commonmode current measurements, to complete radiated and conducted emission measurements and immunity testing. These experiments not only facilitate students' understanding of fundamental electromagnetic field concepts by using applications from electromagnetic compatibility, but they also demonstrate the relevance of electromagnetics principles for designing different circuits and communication systems. The lab manual is available on the web site http://engineering.sfsu.edu/nsf/. Each lab is self-contained and starts with the objectives. It continues with a brief theoretical background, recommended test equipment, detailed measurement procedures and references.

The extensive EMC-related curriculum and a state-of-the-art lab resulted not only in the improvement of the student satisfaction and learning experience, but also gave rise to a high quality undergraduate research program. Over 30 students have been involved in research projects ranging from EM field coupling onto vehicle wiring to cross-talk on printed circuit boards. A number of student and regular papers have been presented at the IEEE AP-S/URSI and EMC Symposia as well as at the meetings of the SCV EMC Society. The first place in the 1999 IEEE Region 6 Student Paper competition and second place in the 2000 WESCON Student Paper competition are a testament of the program quality. The SFSU students are very highly regarded by the local companies due to their EMC training and proficiency. Also, the SCV EMC Society has established a scholarship endowment to support outstanding SFSU students in the $\mathrm{EM} / \mathrm{EMC}$ area.

The next section gives a brief overview of the implementation of the EMC concepts in the EE curriculum. The following sections describe the details of the implementation. The last section addresses the student perception of the revised curriculum and the conclusions.

II. Overview of the Implementation of the EMC Principles in the EE Curriculum

The author's objective was to enhance the existing electromagnetics and communications curriculum by 
- incorporating electromagnetic compatibility concepts and measurement principles more fully into the undergraduate curriculum

- providing students with the invaluable practical experience in using state-of-the-art EMC equipment

- providing students with the opportunity to pursue their regular or senior design projects that include design, implementation, simulation and EMC testing.

This enhancement facilitated the teaching of electromagnetic fields and waves concepts essential for designing circuits and systems with current technology. The students learned to apply the EMC principles early on in the design by integrating their knowledge from a variety of courses: electronic circuits, systems analysis, electromagnetics and communications. The students combined the critical thinking with the practical training while doing the required design projects.

Three existing courses were overhauled and a new course developed. The existing courses, the electromagnetic (EM) field sequence consisting of Introduction to Engineering Electromagnetics (IEE) and Electromagnetic Waves (EMW), and Communications Systems (COS), are by nature highly mathematical and the students found them very difficult. This was especially case with the first course of the EM sequence because of the complete lack of the experimental component. The retention of knowledge acquired in this course was low and resulted in a prolonged review in the second EM course. The students also felt that this was a repetition of a physics course and could not see much relevance to digital circuits. This prompted the author to move the basic transmission line theory from the second to the first course in the EM sequence and compress the static field portion of the course focusing it on calculation of capacitance and inductance of different transmission lines. The frequency- and time-domain analyses were related to analog and digital circuit applications. Also, four EMC-related experiments and a design project were incorporated as described in a consequent section. In return, the second EM course could devote more time to coupled transmission lines important, for example, for crosstalk on PCBs, as well as their numerical modeling. In this case four EMC experiments were added to the existing exercises on standing wave patterns on transmission lines and waveguides, and antenna pattern measurements. The required design project was improved with the use of sophisticated numerical modeling software and incorporation of the EMC design principles.

The Communication Course already had a number of experiments related to oscillators, power amplifiers, amplitude and frequency modulation and demodulation. The author added an experiment on spectra measurement with emphasis on the EMC consequences of digital pulse rise and fall times.

Since the EMC is the integral part of circuit and system design it is necessary to incorporate it more fully in the curriculum an advanced EMC course that will prepare students for challenges of the current and future technologies was established. This course includes four experiments and a design project. 
The next four sections briefly describe the scope of each course with particular emphasis on the newly introduced EMC topics, experiments, and projects.

\section{Introduction to Engineering Electromagnetics}

The introductory course, IEE, covers static electric and magnetic fields with emphasis on capacitance and inductance, time-varying electromagnetic fields and Maxwell's equations, and the basic transmission line theory. In order to facilitate the comprehension of the underlying electromagnetic theory and show its relevance to engineering practice four EMC related experiments have been incorporated into this course: (1) E- and H-field Probing of Noise Sources, (2) Measuring of Capacitance and Inductance of Cables, (3) Faraday's Law and Inductance, and (4) Time and Frequency Behavior of Transmission Lines. Some of the experiments are similar to the exercises described in ${ }^{1,2,3}$.

The first experiment is performed after the students have mastered the concepts of electric and magnetic fields in the lecture portion of the course. They first measure quasi-static fields in the vicinity of simple sources such as straight conductors and rectangular and circular loops and relate them to the theoretical predictions. For that purpose they measure currents in conductors by using current probes and oscilloscope. Then they probe more complicated noise sources such as power supplies, power cables, signal cables and digital circuits. For these measurements they use a set of commercial $H$ - and E-field probes, preamplifier, oscilloscope and/or spectrum analyzer.

The second experiment is scheduled after the capacitance and inductance have been covered in the lectures. Students perform their measurements on twin, coaxial, and twisted shielded pair cables using an RLC meter. By measuring the capacitance between signal lines and between signal lines and shields they can determine the mutual capacitance. This exercise also helps them integrate the circuit approach with the field theory.

In the third experiment students study Faraday's law as one important proof of Maxwell's equations which most of them find very difficult to understand. This exercise also explains the concept of mutual inductance and its dependence on loop areas, distance between the loops and their orientation. A function generator, an oscilloscope and RLC meter are used for measurements.

The objective of the fourth exercise is for students to understand and explore time-domain and frequency domain behavior of transmission lines. This experiment is performed after the fundamentals of transmission line theory have been covered and consists of two parts. In the Part A students carry out frequency-domain measurements of input impedance of short-circuited and open-circuited transmission line in order to find characteristic impedance. In Part B students use time-domain measurements to observe propagation of digital pulses on transmission lines. For this experiment they use a vector network analyzers, spectrum analyzer, pulse generator, and digital oscilloscope.

Finally, the students have to design a microstrip line and use PSPICE to explore multiple reflections of digital pulses on it. 


\section{Electromagnetic Waves}

This course covers non-ideal behavior of components, coupled transmission lines, plane waves, waveguides and antennas. Again, the material presented is highly mathematical and the students find it very difficult. A related lab course already included experiments on standing wave patterns on transmission lines and waveguides, and antenna radiation pattern. The following EMC experiments were added: (1) High-frequency Component Modeling, (2) Cross-talk in Cables, (3) Surface and Common-mode Current Measurements, and (4) Radiated Emission Measurements.

The first experiment helps students understand the frequency dependent nature of real resistors, capacitors, inductors, and ferrite beads. They first learn how to develop impedance models (equivalent circuits) for these real components that take into consideration a number of different parasitic effects. A network analyzer is then used to measure the impedance as a function of frequency. PSPICE is employed to calculate the impedance of the equivalent model and the numerical data are compared with the measurement results.

The objectives of the second experiment are: (1) to help students understand the mechanism of crosstalk in which the electromagnetic fields of electrical signals on one pair of wires in a cable bundle couple to and induce signals in another pair of wires; and (2) to investigate the factors that influence the coupling and methods for reducing the crosstalk. For this experiment students use a function generator and an oscilloscope.

Experiment (3) explores surface currents on shields and common mode currents on power cables that are among the most important sources of EM noise. Students first measure these currents using a set of surface and clamp-on current probes, an oscilloscope and a function generator. Then they measure the EM fields with a set of $H$-and E-field probes, a preamplifier and a network analyzer, and try to correlate them to the currents.

Toward the end of the semester, when the radiation theory has been covered in the lectures, students perform radiated emission measurements. A G-TEM cell was selected for these measurements since it is compact - certainly smaller and cheaper than a semi-anechoic chamber, and more convenient than the open area test site (OATS). It also provides a safe working environment for students since the EM fields are contained within the cell. A spectrum analyzer was connected to the GTEM cell and a $P C$ was used to collect the measurements via an automated GTEM software program. A preamplifier enabled students to perform measurements of low-level radiations. Emissions from different devices such as PC boards, filters, power supplies, cellular phones, etc., were investigated. A computer program has been developed to provide correlation of the GTEM data to the standard OATS measurements ${ }^{4,5,6}$.

The required computer project was also revamped by using a sophisticated EM modeling software (FEM or FDTD) to solve for capacitance and inductance of single and coupled transmission line. Field patterns of different line configurations are also explored. 


\section{Communication Systems}

This course covers Fourier Series and Fourier Transform, amplitude, frequency, and pulse modulation, and related communications systems. The existing lab covered a few standard experiments (oscillators, amplifiers, amplitude modulators and demodulators, and frequency modulators and demodulators). A new Signal Spectra Measurement experiment that is EMC related has been incorporated in this course. Signal spectra is a very difficult topic for students since it is based on highly mathematical concepts such as Fourier Series and Fourier Transform. It is extremely useful for students to be able to visual these concepts and to realize that the spectrum analyzer does show what the theory predicts. Signal spectra of $\mathrm{CW}$, rectangular, trapezoidal and triangular pulses are examined in this experiment. The objectives of this experiment are: (1) to investigate the effect of pulse rise/fall times on the frequency (spectral) content of typical periodic clock signals; (2) to show that pulses having short rise/fall times have frequency spectral content that extends to higher frequencies than do pulses having longer rise/fall times; and (3) to investigate the effect of lead inductance on the performance of circuit elements such as capacitors.

\section{Electromagnetic Compatibility}

A new Electromagnetic Compatibility (EMC) course has been developed based on the model of an undergraduate electromagnetic compatibility course established by Professor Clayton Paul at the University of Kentucky ${ }^{1}$. The course was first pilot-tested on a small group of students under the sponsorship of the Santa Clara Valley EMC Chapter. The course covers two main aspects of electromagnetic compatibility, emissions and susceptibility, and focuses on the design practices for electromagnetic compatibility. Emission is the ability of a device to produce electromagnetic interference and with respect to the coupling path can be radiated or conducted. Due to different mechanisms of these emissions they require different type of measurements and have separate government imposed requirements. Two exercises are developed to accommodate for these differences: (1) Radiated Emission, and (2) Conducted Emission. With the increase of clock speeds and the miniaturization of the circuits, the immunity issue is getting more and more important. Experiment (3), Radiated Immunity, addresses this important practical. We also revisited the experiment on (4) High-frequency Component Modeling. A design project was incorporated in the EMC course as well.

Experiment (1) is similar to the radiated emission measurements in the EMW course. The same test set-up configuration is used: a G-TEM cell with an isotropic probe, a preamplifier, and a spectrum analyzer, controlled by the GTEM software program. In the Experiment (2) a LISN (line impedance stabilization network) and a transient limiter are added for conducted emission measurements. The new laboratory equipment allows students to perform radiated and conducted emission experiments using state-of-the-art equipment.

In the Experiment (3) a different G-TEM cell configuration is used. An incident field in the $G$ TEM cell is produced by a signal generator and a power amplifier. An isotropic probe, and spectrum analyzer connected to the $P C$ are used for the automated measurements. The students follow the FCC guidelines for immunity testing. 
The students also have had a semester long project to design a circuit or a system that would pass the Federal Communications Commission (FCC) requirements. This was a culminating experience in combining knowledge from all the previous circuits and electronics courses with the EMC concepts. Students could propose a project or choose one from the list compiled by the instructor. Some of the examples were: a simple digital circuit with a single clock, switching power supplies, and PC motherboards. The students were required to (a) design, (b) simulate, (c) test for compliance, and (d) redesign if necessary. They worked in teams of 2-3 members. They tested their selected projects for radiated and conducted emissions early on in the design cycle. It was quite a revelation to them that a system could operate properly but still was unacceptable in the real world because it could not pass the FCC requirements. The early testing gave them enough time to apply different EMC "remedies" in the case that their design failed, and to repeat the measurements a few times during the design process if necessary.

\section{Conclusions}

The EMC concepts have been implemented throughout the EE curriculum at San Francisco State University by overhauling two EM courses, improving a communication system course, and introducing a new EMC course. With the help from the NSF, industry partners, and the Santa Clara Valley EMC Society, a new EMC lab was established to provide the experimental component necessary for understanding of the complex EM and EMC principles and applications. The student learning experience and overall course satisfaction has been significantly improved. Before the EMC applications and experiments were implemented, the students comments about the courses ranged from: "Very mathematical, need more examples of real life problems" and "Mostly a rehash of the EM physics, with only a few new concepts added" to "I wish we had some direct application we could use as lab (experiment)" and "Should include more practical stuff rather than so much theory." The revised courses received evaluations such as: "I learned a lot of electromagnetics material in this course; Fun; Helpful and intense; Useful, practical; This was a tough course, but all the visual aids, learning tools and experiments are helpful in better understanding of the material." The students also learned to apply the EMC principles early on in the design by integrating their knowledge from a variety of courses: electronic circuits, systems analysis, electromagnetics and communications. This integrated EMC education prepares students for design challenges of the future by providing them with hands-on experience in dealing with the EMC problems and solutions.

\section{Bibliography}

[1] Paul, C. R., "Establishment of a university course in electromagnetic compatibility (EMC)," IEEE Trans. Education, vol. 33, Feb. 1990, pp.119-128.

[2] Drewniak, J. L., Hubing, T.H., Van Doren, T.P., and Sha, F., "Integrating electromagnetic compatibility laboratory exercises into undergraduate electromagnetics," Proc. of IEEE Int. Symp. on EMC,pp.35-40, 1995.

[3] Ott, H.W., and Paul, C.R., "Experiments and Demonstration in Electromagnetic Compatibility," Eds. Education Committee of the IEEE Electromagnetic Compatibility Society, 1992.

[4] Wilson, P., "On simulating OATS near-field emission measurements via GTEM cell measurements," Proc. of 1993 EMC Int. Symp. Electromagnetic Compatibility, pp.53-57, Dallas, TX, 1993.

[5] Osburn, D. M., and Bronaugh, E. L., "Advances in GTEM to OATS correlation models," Proc. of 1993 EMC Int. Symp. Electromagnetic Compatibility, pp.95-98, Dallas, TX, 1993. 
[6] Wilson, P., Hansen, D., and Koenigstein, "Simulating open area site emission measurements based on data obtained in a novel broadband TEM cell," IEEE Proc. of 1989 Symp. on Electromagnetic Compatibility, pp.171177, Denver, CO, 1989.

\section{ZORICA PANTIC-TANNER}

Zorica Pantic-Tanner is a Professor of Electrical Engineering and Director of the School of Engineering and Computer Science at San Francisco State University. Her teaching and research interests are in the areas of applied electromagnetics such as: electromagnetic compatibility, analysis and design of passive microwave circuits and printed circuit boards, antenna design, and calculation of optical properties of biological macromolecules. She has published over 70 papers in these areas. She has established a Center for Applied Electromagnetics using an NSF grant and generous equipment donations from the SFSU industry partners. Dr. Pantic-Tanner is a Senior Member of the IEEE Society. She has served as a Secretary, Treasurer, Vice Chair, and Chair of the Santa Clara Valley Chapter of the IEEE EMC Society. She is currently the Vice Chair of the Numerical Modeling Committee and a member of the Education Committee of the IEEE EMC Society. She also serves as the Board Member of the ASEE PSW Section. Dr. Pantic-Tanner received her B.S., M.S., and Ph.D. degrees in Electrical Engineering from the University of Nis in Yugoslavia in 1975, 1978, and 1982, respectively. 